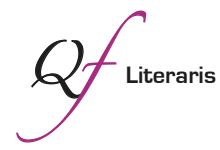

\title{
Revisión crítica de la edición elaborada por L. P. Gachard de Lettres de Philippe II a ses filles les Infantes Isabelle et \\ Catherine: écrites pendant son voyage en Portugal (1581-1583) $\mathrm{y}$ trayectoria de posteriores ediciones a lo largo del siglo $\mathrm{XX}$
}

\author{
Critical revision of the edition by L. P. Gachard of Lettres de Philippe II \\ a ses filles les Infantes Isabelle et Catherine: écrites pendant son voyage \\ en Portugal (1581-1583) and evolution of later editions in the 20th century
}

\author{
Miriam N. Bucuré
}

Universitat de València. miriam.bucure@uv.es

Recibido: 09/05/2017. Aceptado: 04/09/2017

Resumen: Basándonos en los documentos editados por L. P. Gachard en Lettres de Philippe II a ses filles les Infantes Isabelle et Catherine: écrites pendant son voyage en Portugal (1581-1583), pretendemos revisar y analizar críticamente la metodología empleada por el historiador belga en la edición de textos en la segunda mitad del siglo XIX. Esta documentación, publicada en 1884, fue reeditada alrededor del 1943 por Luisa Elena del Portillo Díaz y en 1988 por el historiador español Fernando Bouza. Es nuestra intención revisar la edición de Gachard a la luz de las nuevas ediciones y examinar de esta forma la trayectoria y los progresos, si los hubiere, en la edición de un mismo texto a lo largo de un siglo.

Palabras clave: edición de textos; edición diplomática; edición divulgativa; epistolario; Felipe II.

\begin{abstract}
Grounding on the documents Lettres de Philippe II a ses filles les Infantes Isabelle et Catherine: écrites pendant son voyage en Portugal (1581-1583) edited by L. P. Gachard, we expect to revise critically the methodology used for the editions of the texts by the Belgian historian in the second half of the XIX century. This documentation was published in 1884 and reprinted by Luisa Elena del Portillo Díaz around 1943 and then by the Spanish historian Fernando Bouza in 1988. It is our intention to revise Gachard's edition in the light of new editions, paths and progresses, if any, in the edition of one text over a century.
\end{abstract}

Keywords: text editing; diplomatic editing; informative editing; collection of letters; Felipe II.

/) Bucuré, Miriam N. 2017. "Revisión crítica de la edición elaborada por L. P. Gachard de Lettres de Philippe II a ses filles les Infantes Isabelle et Catherine: écrites pendant son voyage en Portugal (1581-1583) y trayectoria de posteriores ediciones a lo largo del siglo XX". Quaderns de Filologia: Estudis Literaris 22: 41-55. doi: 10.7203 /qfed.22.11251 



\section{Introducción}

A partir de las cartas de Felipe II a sus hijas Isabel Clara Eugenia y Catalina Micaela editadas por el historiador belga, de origen francés, Louis Prosper Gachard en Lettres de Philippe II a ses filles les Infantes Isabelle et Catherine: écrites pendant son voyage en Portugal (15811583), pretendemos revisar y analizar críticamente la metodología empleada por el historiador en la edición de textos en la segunda mitad del siglo XIX y estudiar la trayectoria de las ediciones que, con posterioridad, se realizaron a lo largo del siglo XX.

Esta primera edición de las cartas, treinta y cuatro en total, se publicó en 1884 y fue muy bien recibida por los historiadores que combatían la leyenda negra de Felipe II, poniendo de relieve la imagen de padre cariñoso, devoto y entregado a su familia que ofrecía de sí mismo en las cartas. A principios de 1940 Luisa Elena del Portillo Díaz reedita estas cartas en Madrid con el título Cartas de Felipe II a sus hijas ${ }^{1}$, y en 1988 sale a la luz la primera edición de un total de ciento treinta y tres cartas que el monarca español escribe a sus hijas, entre las cuales las treinta y cuatro editadas por L. P. Gachard, de manos del historiador español Fernando Bouza ${ }^{2}$.

Nuestra intención es revisar la edición de Gachard a la luz de las nuevas ediciones y examinar de esta forma los progresos, si los hubiere, en la edición de un mismo texto a lo largo de un siglo.

La edición de Gachard fue ampliada, en un primer momento, por Erika Spivakovsky en 1975. En su obra el historiador belga deja constancia clara de otras noventa y una cartas que el monarca español escribe en exclusiva a su hija la infanta Catalina Micaela, después que esta contrajera nupcias con Carlo Emanuele ${ }^{3}$, duque de Saboya en marzo

\footnotetext{
* El presente trabajo forma parte del proyecto de investigación Las mujeres en la casa de Austria (1526-1567). Corpus Documental (FFI2014-52227-P).

${ }^{1}$ No hay manera de conocer la fecha exacta de esta publicación. El ejemplar consultado, editado por Lepanto, carece de datación. En el catálogo de la Biblioteca Nacional hay dos entradas a la obra, fechadas en 1943[?] y 1944[?].

${ }^{2}$ Una segunda edición, corregida y aumentada, fue publicada en 1998. Para el presente estudio hemos utilizado esta segunda publicación: Bouza, 1998.

${ }^{3}$ Hijo de Emanuele Filiberto y de Margherita di Valois, Carlo Emanuele nace en el castillo de Rivoli, en Piamonte, el 12 de enero de 1562. Es duque de Saboya desde 1580 a 1630. Sobre su gobierno en Saboya, cfr. Castronovo, 1977: 326-340.
} 
de $1585^{4}$. Gachard no edita dichas cartas, simplemente las presenta en apéndice, ofreciendo un simple listado de ellas donde consta la datación y el lugar donde fueron escritas.

Pero volviendo a las cartas objeto de nuestro estudio, es importante recalcar que ponen de manifiesto una imagen hasta entonces desconocida de Felipe II. Ya en 1876 Juan Pérez de Guzmán había escrito un artículo en Revista contemporánea titulado "El lado amable de un rey severo" con el objetivo de reivindicar la figura del rey católico:

Feliz yo si consigo mediante este pálido ensayo atraer entre mis lectores alguna atención simpática hácia el monarca sobre cuya memoria tanta saña ha derramado el espíritu crítico de los cien últimos años (Pérez de Guzmán, 1876: 77).

Ocho años más tarde, Louis Prospere Gachard editó estas treinta y cuatro cartas que escribió Felipe II a sus hijas, las infantas Isabel Clara Eugenia y Catalina Micaela entre 1581 y 1583, durante la estancia del monarca en Portugal con motivo de la anexión de ese reino a la corona española ${ }^{5}$. Estas cartas de carácter privado permiten al lector construir una nueva imagen más cercana, más amable del monarca y atenúan hasta hacer desaparecer la ominosa e infausta imagen del que era llamado "demonio del mediodía" por la crítica extranjera más severa. Fernando Bouza en la introducción a su obra habla de lo consciente que era Felipe II de la propia capacidad para crear "memoria y también olvido de sus hechos, de sus decisiones, de su espíritu y de su propia majestad". Agrega, además, que el monarca católico pretendió determinar no solo el modo en que quería ser conocido, sino también cómo ser recordado (Bouza, 1998: 5-6). No tuvo en cuenta el rey católico que estas cartas, pese a su intención y orden expresa de ser "olvidadas", serían conser-

\footnotetext{
${ }^{4}$ Estas cartas comprenden el período entre los esponsales de Catalina Micaela en 1585 hasta un año antes de su muerte en 1596. Spivakovsky no edita noventa y una cartas, sino noventa y tres. En el prólogo de su obra, la autora especifica que la lista que ofrecía Gachard se presentaba con "ciertos errores y algunas omisiones" (Spivakovsky, 1975: 10). Así pues, una vez corregidos los errores y solventadas las omisiones, las cartas que el monarca español remite a su hija la duquesa de Saboya suman noventa y dos. A este corpus documental la autora añade una carta más escrita el 16 de noviembre de 1594 desde San Lorenzo, pero firmada por la infanta Isabel Clara Eugenia.

5 "La Correspondance que nous publions commence un peu après l'arrivée du Roi à Thomar; elle se continue jusqu'à son retour en Castille”, cfr. Gachard, 1884: 19.
} 
vadas por su segunda hija Catalina Micaela. En la carta que escribe a las infantas desde Lisboa el 30 de julio de 1582, el monarca dice al respecto: "A las demás cartas vuestras, por ser ya viejas, acuerdo de no responder, sino quemarlas, por no cargar más de papeles [...]" (Gachard, 1884: 184).

En ningún caso, tanto las misivas que él componía como las que recibía de sus hijas, se consideraron por entonces dignas de ser conservadas en el Archivo Real de Simancas. Sin embargo, la infanta Catalina Micaela las llevó consigo al ducado de Saboya tras la boda (Spivakovsky, 1975: 10) y es por ello que estas, junto con las editadas por Spivakovsky y otras siete hológrafas de Felipe II que menciona y edita Bouza en su obra, las custodia el Archivio di Stato di Torino.

\section{Las obras de Gachard, de Portillo Díaz y de Bouza}

En esta sección referiremos el modo en que estos tres estudiosos han presentado la cuestión de las cartas en sus obras, señalando las diferencias y similitudes que estas tres obras revelan.

\subsection{4: la obra de Louis Prosper Gachard}

En el año 1867 el historiador belga localizaba estas cartas en el por entonces llamado Archivio Reale di Torino.

Da cuenta de ello en la Introduction a su obra, en el primer epígrafe que titula Observations Préliminaires. A este le siguen otros cinco epígrafes en los que describe minuciosamente el marco histórico que abarca el período desde 1578, año en el que fallece don Sebastián I de Portugal hasta el 1633, año de la muerte de la infanta Isabel Clara Eugenia. El último epígrafe de la Introduction lo dedica a los variados temas que se tratan en las misivas. Antes de proceder a la transcripción de las cartas añade dos apéndices: el primero, al que nos hemos referido anteriormente, bajo el rótulo: "Lettres de Philippe II a l'infante Catherine, duquesse de Savoie, conservées dans les Archives Royales de Turin", informa de la existencia de las cartas que el monarca escribe en exclusiva a la infanta Catalina Micaela a partir de 1585; el segundo refiere los objetivos que tenía Felipe II sobre los asuntos de Francia y lo que pretendía obtener en los Estados Generales, en una traducción al francés del documento: "El intento que tiene Su Magestad en las cosas 
de Francia, y ha mandado y es servido que se procure encaminar en los estados generales", firmado en Madrid en enero de 1592 (Gachard, 1884: 43-44 y 74-80).

Esta primera parte de la obra de Gachard se cierra con los dos apéndices. En la segunda parte se halla la edición de las cartas acompañadas todas ellas por una traducción en francés.

\subsection{3[?]: la obra de Luisa Elena del Portillo Díaz}

Calcando el esquema de la obra de Gachard, Portillo Díaz presenta la edición de las cartas precedida por un prólogo en el que refiere, mayormente, datos históricos. En el primer párrafo del mismo, la autora justifica su trabajo: considera que "si bien en España [las cartas] fueron conocidas y estudiadas por los eruditos, no llegaron a tener una resonancia y eco popular" y señala la importancia de dar a conocer esta documentación "al gran público" (Portillo Díaz, 1943 [?]: 7) con el fin de crear una nueva imagen del monarca español a partir del descubrimiento de esa nueva dimensión más benigna, más íntima, más afable.

Tras el prólogo, en que también describe detalladamente los temas abordados en las cartas, las edita. A continuación, y sin anotar, en apéndice, añade la transcripción de cinco documentos, cuatro de ellos extraídos de las Memorias de Fray Juan de San Gerónimo ${ }^{6}$, a excepción de uno proveniente de la Real Academia de la Historia ${ }^{7}$.

Desconocemos los motivos por los que la autora escoge tales escritos y no otros que podrían resultar tan pertinentes como estos para ilustrar su obra.

\footnotetext{
${ }^{6}$ Documento 1 - Partida que hace el Rey Don Felippe nuestro Señor, a tomar posesión del reino de Portugal, con la resolución que dió la facultad de Teología de la Universidad de Alcalá acerca de la prosecución del derecho que S. M. el Rey Don Felippe nuestro Señor, tiene a los reinos de la corona de Portugal. Documento 2 - El notable catarro que anduvo en España. La enfermedad peligrosa del Rey Don Felippe nuestro Señor. La muerte de la Reina Doña Ana nuestra Señora. Documento 3 - Relación de la orden que se tuvo en el juramento del Rey Don Felippe nuestro Señor por los tres Estados de los reinos de Portugal, domingo 16 de abril de 1581 años. Documento 5 - Venida del Rey Don Felippe nuestro Señor a Castilla desde Portugal y por este Monasterio. Queda por Gobernador el Príncipe Cardenal D. Alberto sobrino de S. M. (Portillo Díaz, 1943 [?]: 107-123 y 128-131).

${ }^{7}$ Documento 4 - Relación de la entrada que hizo S. M. en Lisboa el día de Sant Pedro, que se contaron 29 de junio 1581 (Portillo Díaz, 1943 [?]: 124-127).
} 
Acerca de la documentación presentada en el apéndice, Portillo Díaz tiene a bien dar la localización precisa de la misma: respecto a las $\mathrm{Me}$ morias de Fray Juan de San Gerónimo, señala que procede de la Biblioteca del Escorial. Ms.: K-I-7; el escrito procedente de la RAH está registrado como: Jesuitas, tomo 150 de papeles varios. La atenta lectura de la edición de las cartas y de esta documentación nos ha llevado a pensar que la autora no trabaja en base a los textos originales, sino que se limita a transcribir documentos ya editados.

\subsection{8: la obra de Fernando Bouza}

Entrados ya casi en el siglo XXI, Fernando Bouza presenta una reedición de las Cartas de Felipe II a sus hijas que había publicado en 1988. En este caso, el historiador español reúne el epistolario completo de Felipe II y las infantas. No solo presenta las treinta y cuatro "cartas portuguesas", editadas por Gachard y posteriormente por Portillo Díaz; sino que añade las noventa y dos enviadas a la infanta Catalina Micaela a Saboya que en 1975 editara Spivakovsky; y siete cartas más, también custodiadas en el Archivio di Stato di Torino.

En la introducción Bouza examina la figura de Felipe II y analiza el carácter de las cartas. Presenta, además, las circunstancias históricas en donde se desarrollan los hechos; describe los escenarios habituales por los que se pasea Su Majestad; menciona los temas tratados en las cartas y presenta a los personajes que las pueblan.

Tras la introducción, expone una tabla de correspondencia de las cartas hasta el momento editadas por Gachard y por Spivakovsky. Añade otra columna en la que refiere la correspondencia mantenida entre el monarca español y duque de Saboya según la edición de Giovanna Altadonna de $1986^{8}$.

A continuación, edita las ciento treinta y tres cartas. Para finalizar, antes de indicar la bibliografía y el índice de personas y lugares, ofrece tres cuadros genealógicos, dos referidos a la Casa de Austria en sus ramas española y austríaca respectivamente y uno referido a la Casa de Saboya.

\footnotetext{
${ }^{8}$ Bouza no incluye en su obra la edición de la correspondencia entre Felipe II y Carlo Emanuele di Savoia. Acerca de dicha relación epistolar, cfr. Altadonna, 1986: 137-190. Antes de presentar la edición de estas misivas, la autora tiene a bien analizar tanto el estilo como la grafía del monarca español.
} 


\section{Las ediciones de las "cartas portuguesas" a través de los siglos}

\subsection{Criterios de edición y sistema de notas}

Tanto Gachard como Portillo Díaz han realizado una edición diplomática de los documentos, respetando todas las particularidades que presenta cada una de las cartas en lo que respecta a grafía, a morfología y a sintaxis.

Sin embargo, nos atreveríamos a conjeturar que la autora española ha transcrito las misivas desde la obra de Gachard. Nuestra suposición se fundamenta en que varios de los errores de transcripción que comete Gachard en su obra se encuentran también plasmados en la obra de Portillo Díaz?.

Gachard no hace referencia alguna a los criterios de edición, simplemente justifica la traducción al francés de las misivas en la introducción ${ }^{10}$. Tampoco Portillo Díaz menciona criterios de edición.

Bouza, en cambio, ha optado por modernizar la grafía y la puntuación. Ha intervenido, además, con pequeños cambios sobre la sintaxis y la morfología "para aclarar ciertas frases que, de lo contrario hubieran podido resultar de difícil comprensión" (Bouza, 1998: 25). El historiador español tiene a bien aclarar que la edición de las primeras veintiocho cartas se ha realizado "a partir de la comparación de la edición de Gachard de 1884 con los originales turineses" (Bouza, 1998: 25). Estos son los criterios referidos por Bouza que dan como resultado una edición de corte histórico-divulgativo.

La mayor parte de las anotaciones que se observan en la edición de 1884 son de carácter histórico y/o informativo y surgen de la traducción al francés, nunca de la transcripción en castellano.

En toda la obra se verifican solo tres notas de tipo filológico; la primera la encontramos en la carta número XIV, remitida desde Lisboa el 29 de enero de 1582: "El que canta con los órganos deve ser ..........., que conocerá mejor my hermana" (Gachard, 1884: 139). En la transcripción

\footnotetext{
${ }^{9}$ A modo de ejemplo, el verbo "posar" que reportan ambos estudiosos en lugar de "parar" en la correcta lección de Bouza en la siguiente frase de la carta XXXII: "Y así, por rodear algo, y aver de posar en algunos lugares".

${ }^{10}$ No solo justifica la traducción, sino que, además, se excusa por posibles errores: "Je réclame l'indulgence pour les erreurs que je puis avoir commises". Cfr. Gachard, L.P. 1884: Lettres de Philippe II..., op. cit., p. 69.
} 
castellana el estudioso se limita de dejar un espacio en blanco señalado con varios puntos suspensivos, mientras que de la traducción al francés se desprende una nota a pie de página: "mot illisible" (Gachard, 1884: 143). Portillo Díaz hace caso omiso de la laguna que indica con tres puntos suspensivos (Portillo Díaz, 1943 [?]: 59). Es el historiador español quien completa la información: "El que canta con los órganos debe ser Talamantes, que conocerá mejor mi hermana" (Bouza, 1998: 69 , n. 124).

La segunda nota filológica que hallamos en la edición de Gachard se presenta en la carta XXV y hace referencia a la ausencia de un folio. La frase de la que se desprende la nota filológica es la siguiente:

Y no cay en verlo de los aposentos altos, sino del baxo donde posava, aunque creo que del uno ny del otro no se vía sino la llama del fuego que hera muy grande, por ser la casa no Se parece de allí [...] (Gachard, 1884: 192-193).

Garchard interrumpe la transcripción señalando la falta del folio con unos cuantos puntos suspensivos como en el caso anterior, pero esta vez anota a pie de página:

Ici finit la deuxième page de la lettre qui forme une feuille. II doit y manquer une feuille ou un feuillet intercalé. La troisième page commence par se parece de allí (Gachard, 1884: 193, n. 1).

Portillo Díaz compone prácticamente la misma nota filológica: "parece faltar la hoja siguiente de la carta" precisando inmediatamente "en opinión de Gachard"11.

El historiador español argumenta al respecto que no se trata de la falta de un folio como supone Gachard sino de un problema de interpretación del verbo "parecer" que está por "ver", por "divisar". De manera que el párrafo en la edición de Bouza dice así:

Y no caí en verlo de los aposentos altos, sino del bajo donde posaba, aunque creo que del uno ni del otro no se veía sino la llama del fuego

\footnotetext{
${ }^{11}$ Este es otro de los ejemplos a raíz de los cuales nos atrevemos a conjeturar que la autora publica las cartas de Felipe II a sus hijas en base a la edición de 1884 (Portillo Díaz, 1943 [?]: 88).
} 
que era muy grande, porque la casa no se parece $^{12}$ de allí y la Puerta creo [...] (Bouza, 1998: 93).

En esta misma misiva, ya casi al final, hay un pasaje muy interesante en la edición de Gachard -y, por ende, también, en Portillo Díaz-, en el que transcribe la palabra "hieromóvoces" que dice así:

Muy bien es que vuestro hermano no tenga myedo, como decís, vos la menor, y no creo que le tubiera de los diablos de la procesión, porque venían buenos y víanse de lexos, y mas parecían cosas di hieromóvo$c e s^{13}$ que no diablos; y cierto que heran buenos, pues no heran verdaderos (Gachard, 1884: 193 y Portillo Díaz, 1943 [?]: 88).

En la transcripción en castellano no se apunta ninguna nota a pie de página. En el texto en francés, en cambio, deja un espacio en blanco señalado, como de costumbre, con varios puntos suspensivos, y anota:

Je n'ai pu, malgré tous mes efforts, trouver une traduction satisfaisante des mots cosas de hieromóvoces, et je me suis vainement adressé, pour en avoir l'explication, à des personnes auxquelles la langue castillane est familiére (Gachard, 1884: 196).

El párrafo en la lección de Bouza:

Muy bien es que vuestro hermano no tenga miedo como decís vos, la menor, y no creo que le tuviera de los diablos de la procesión, porque venían buenos y veíanse de lejos y más parecían cosas de Jerónimo Bosco $^{14}$ que no diablos y cierto que eran buenos, pues no eran verdaderos (Bouza, 1998: 93).

En la carta XXVI enviada desde Lisboa el 1 de octubre de 1582 hallamos la tercera nota filológica: "No creo que podrá dexar de sentir don Diego de Cordova la muerte de ..........."15. Portillo Díaz anota la falta de información como "palabra ilegible" (Portillo Díaz, 1943 [?]: 90). En

\footnotetext{
${ }^{12}$ La cursiva es nuestra.

${ }^{13}$ La cursiva es nuestra.

${ }^{14}$ La cursiva es nuestra.

${ }^{15}$ En este caso como en los anteriores el historiador belga anota la ausencia del nombre con varios puntos suspensivos y anota a pie de página "nom illisible" (Gachard, 1884: 198).
} 
la lección de Bouza se resuelve la cuestión. El nombre que falta en las ediciones precedentes es el de doña Ana María Laso de Castilla, consorte del señor de Almúñar, don Diego Fernández de Córdoba (Bouza, 1998: 94-95, nn. 206 y 207).

En la edición de la correspondencia de Felipe II que realiza el historiador español en 1998, se combinan a pie de página las notas históricas y las filológicas. La edición se enriquece, además, con notas que completan la información de las cartas en las que se transcriben párrafos de documentos custodiados en bibliotecas y archivos europeos varios.

Hay una nota filológica que redacta Bouza que es digna de mención. Se refiere a la fecha de la última carta, remitida desde San Lorenzo. En la misiva consta simplemente "martes noche" y no "abril de 1583", tal y como lo especifica Bouza. Según la explicación del historiador español, el señalamiento del mes de abril se debe a Gachard (Bouza, 1998: 105-106, n. 239). Sin embargo, al consultar la edición de 1884, hemos notado que, en la traducción al francés, en la parte en que especifica el lugar y la fecha, el historiador belga dice en: "Saint-Laurent, mardi soir (... mars 1583)" y anota a pie de página por qué, según él, la misiva debe ser datada en marzo de 1583 (Gachard, 1884: 227, n. 1).

\subsection{Collatio}

Antes de presentar las diferencias más relevantes entre las tres ediciones nos gustaría aclarar que los tres autores han tenido a bien desarrollar las múltiples abreviaturas que el monarca español ha utilizado al componer las misivas.

A continuación, y a modo de collatio, señalaremos algunas variantes de las tres versiones, donde por $\mathrm{G}$ se entiende la edición de Gachard de 1884; por P, la edición de Portillo Díaz de 1943[?] y por B, la edición de Bouza de 1998.

En primer lugar, mostraremos las diferencias relativas al tratamiento de los números para después ofrecer algún ejemplo de errores de copia entre los cuales las omisiones -tanto homeoteleuton como lagunas-, interpolaciones y trasposiciones.

Respecto a la transcripción de los números, Gachard y Portillo Díaz reproducen las cifras en números arábigos o romanos tal y como se presentan en el texto original; Bouza evita la cifra, prefiriendo escribirlos 
en palabras, exceptuando, claro está, los casos referidos a la datación de cada carta o cuando se refiere a una fecha.

\begin{tabular}{|l|l|l|}
\hline \multicolumn{1}{|c|}{ carta } & \multicolumn{1}{c|}{ G (1884) - P (1943?) } & \multicolumn{1}{c|}{ B (1998) } \\
\hline cc. II/XXVII & 8 días & ocho días \\
\hline c. V & 14 o 15 galeones & catorce o quince galeones \\
\hline c. VII & $X V$ años & quince años \\
\hline c. XXXIII & $X V$ dias & quince días \\
\hline
\end{tabular}

En cuanto a los casos de omisión, también denominada homeoteleuton, hemos notado tres casos en la obra de Portillo Díaz, en las cartas I, $\mathrm{X}$ y XXIX respectivamente.

\begin{tabular}{|l|l|l|}
\hline \multicolumn{1}{|c|}{ carta } & \multicolumn{1}{|c|}{ G (1884) - B (1998) } & \multicolumn{1}{c|}{ P (1943?) } \\
\hline \multirow{4}{*}{ carta I } & $\begin{array}{l}\text { Y de oy en 8 dias creo yo } \\
\text { que embiaré cartas para } \\
\text { ella, con que se havrá } \\
\text { de despachar correo; y } \\
\text { así para entonces podréis } \\
\text { tener escrito }[. . .]\end{array}$ & $\begin{array}{l}\text { Y de oy en 8 días creo yo } \\
\text { que embiaré cartas para } \\
\text { ella; y así para entonces } \\
\text { podreis tener escrito [...] }\end{array}$ \\
\hline \multirow{3}{*}{ carta XXIX } & $\begin{array}{l}\text { y tanto como yo lo estube } \\
\text { los dias que estube en } \\
\text { Belén sin my hermana } \\
{[\ldots]}\end{array}$ & $\begin{array}{l}\text { y tanto como yo lo estube } \\
\text { en Belén sin my hermana } \\
{[\ldots]}\end{array}$ \\
\hline
\end{tabular}

En la carta X, además del caso de omisión, hemos notado la lectura diferente de los verbos "enviar" y "escribir".

\begin{tabular}{|c|c|c|c|}
\hline carta & G (1884) & P (1943?) & B (1998) \\
\hline $\operatorname{carta} X$ & $\begin{array}{l}\text { y respondídole á sus } \\
\text { cartas que os embió; } \\
\text { y deseo mucho saver } \\
\text { que sea ya desem- } \\
\text { barcada; y hizo aquí } \\
\text { tormenta una noche } \\
\text { destas [...] }\end{array}$ & $\begin{array}{l}\text { y respondídole } \\
\text { á sus cartas que } \\
\text { os escribió; } \\
\text { y hizo aquí } \\
\text { tormenta una } \\
\text { noche destas } \\
{[\ldots]}\end{array}$ & $\begin{array}{l}\text { respondídole a sus } \\
\text { cartas que os escribió; } \\
\text { y deseo mucho saber } \\
\text { que sea ya desem- } \\
\text { barcada; e hizo aquí } \\
\text { tormenta una noche de } \\
\text { éstas [...] }\end{array}$ \\
\hline
\end{tabular}


Hemos verificado, además, varios casos de interposición y de omisión de simples palabras. Interesante es el primer ejemplo de omisión, donde se verifican tres lecciones diferentes, que referimos a continuación:

\begin{tabular}{|c|c|c|c|}
\hline carta & G (1884) & $P(1943 ?)$ & B (1998) \\
\hline carta XXXI & $\begin{array}{l}\text { Y ella vio muy } \\
\text { bien ayer el jura- } \\
\text { mento. }\end{array}$ & $\begin{array}{l}\text { Y ella vio muy } \\
\text { bien el jura- } \\
\text { mento. }\end{array}$ & $\begin{array}{l}\text { Y ella vio ayer el } \\
\text { juramento. }\end{array}$ \\
\hline carta XXXII & $\begin{array}{l}\text { Tampoco puedo } \\
\text { responderos agora } \\
\text { a las postreras } \\
\text { cartas }[\ldots]\end{array}$ & $\begin{array}{l}\text { Tampoco puedo } \\
\text { responderos ago- } \\
r a \text { a las postreras } \\
\text { cartas }[\ldots]\end{array}$ & $\begin{array}{l}\text { Tampoco puedo } \\
\text { responder a las } \\
\text { postreras cartas } \\
{[\ldots]} \\
\end{array}$ \\
\hline carta XXX & $\begin{array}{l}{[. . .] \text { y para esto me }} \\
\text { doy toda la priesa } \\
\text { que puedo }\end{array}$ & $\begin{array}{l}{[\ldots] \text { y para esto }} \\
\text { me doy toda la } \\
\text { priesa que puedo }\end{array}$ & $\begin{array}{l}\ldots] \text { y para esto } \\
\text { me doy la prisa } \\
\text { que puedo }\end{array}$ \\
\hline
\end{tabular}

Seguidamente, algún ejemplo de interpolación.

\begin{tabular}{|l|l|l|}
\hline \multicolumn{1}{|c|}{ carta } & \multicolumn{1}{|c|}{ G (1884) - P(1943?) } & \multicolumn{1}{c|}{ B (1998) } \\
\hline carta XXXI & $\begin{array}{l}\text { [...] y yo no puedo ni } \\
\text { quiero agora perder } \\
\text { tiempo en escrivir. }\end{array}$ & $\begin{array}{l}{[\ldots] \text { yo no puedo ni quiero }} \\
\text { ahora perder más tiempo en } \\
\text { escribir. }\end{array}$ \\
\hline carta XXXII & $\begin{array}{l}\text { Y oy he ido yo a su casa } \\
{[\ldots]}\end{array}$ & $\begin{array}{l}\text { Y hoy me he ido yo a su } \\
\text { casa }[\ldots]\end{array}$ \\
\hline carta XXXIII & $\begin{array}{l}{[\ldots] \text { mas luego me pas- }} \\
\text { aré ay por veros y a my } \\
\text { hermana. }\end{array}$ & $\begin{array}{l}{[\ldots] \text { más luego me pasaré }} \\
\text { por ahi por veros y a mi } \\
\text { hermana. }\end{array}$ \\
\hline
\end{tabular}

Respecto a la trasposición, hemos encontrado un único caso. En la carta VII la frase dice así:

\begin{tabular}{|c|c|l|}
\hline \multicolumn{1}{|c|}{ carta } & G (1884) - P (1943?) & \multicolumn{1}{c|}{ B (1998) } \\
\hline \multirow{2}{*}{ carta VII } & $\begin{array}{l}{[\ldots] \text { qu'es gran vegez os }} \\
\text { tener ya tantos años }[\ldots]\end{array}$ & $\begin{array}{l}{[\ldots] \text { que gran vejez es tener }} \\
\text { yantos años }[\ldots]\end{array}$ \\
\hline
\end{tabular}


Para finalizar, presentaremos algunos ejemplos de lecturas diferentes. Veremos cómo las lecciones de Gachard y de Portillo Díaz son similares, siendo discrepante, normalmente, la lección de Bouza.

\begin{tabular}{|c|c|c|}
\hline carta & G (1884) - P (1943?) & B (1998) \\
\hline carta I & $\begin{array}{l}{[\ldots] \text { como vereis en lo que }} \\
\text { va en la cartilla. }\end{array}$ & $\begin{array}{l}{[\ldots] \text { como vereis en lo que }} \\
\text { va en la cajilla. }\end{array}$ \\
\hline carta VI & $\begin{array}{l}{[\ldots] \text { por ser casi las pieças }} \\
\text { de ala a teja vana }[. . .]\end{array}$ & $\begin{array}{l}{[\ldots] \text { por ser casi las piezas }} \\
\text { de acá a teja vana }[\ldots]\end{array}$ \\
\hline carta VII & $\begin{array}{l}{[\ldots] \text { y otras criadas que dex- }} \\
\text { aron de la reina my tía. } \\
\text { Y la hecha de vuestras car- } \\
\text { tas }[\ldots]\end{array}$ & $\begin{array}{l}{[\ldots] \text { y otras criadas que que- }} \\
\text { daron de la reina my tía. } \\
\text { Y la fecha de vuestras car- } \\
\text { tas }[. . .]\end{array}$ \\
\hline carta XXVI & $\begin{array}{l}\text { No querría que se hubiese } \\
\text { pasado el pico de vuestro } \\
\text { hermano }[\ldots]\end{array}$ & $\begin{array}{l}\text { No querría que se hubiese } \\
\text { pasado el pico de vuestra } \\
\text { hermanica }[\ldots]\end{array}$ \\
\hline carta XXVII & $\begin{array}{l}\text { y una hay dentro della con } \\
\text { otras porcelanas. }\end{array}$ & $\begin{array}{l}\text { y una arquilla dentro de } \\
\text { ella con otras porcelanas. }\end{array}$ \\
\hline
\end{tabular}

En la carta XX, hemos hallado tres lecturas diferentes:

\begin{tabular}{|c|l|l|l|}
\hline carta & \multicolumn{1}{|c|}{ G (1884) } & \multicolumn{1}{c|}{$\mathbf{P}(\mathbf{1 9 4 3 ? )}$} & \multicolumn{1}{c|}{ B (1998) } \\
\hline carta XX & $\begin{array}{l}{[\ldots] \text { y my hermana }} \\
\text { y yo hablábamos } \\
\text { oy en ello }[\ldots]\end{array}$ & $\begin{array}{l}\text { [... y my hermana } \\
\text { y yo hablábamos } \\
\text { ay en ello }[\ldots]\end{array}$ & $\begin{array}{l}\text { y...] y mi hermana hablábamos } \\
\text { en ello }[\ldots]\end{array}$ \\
\hline
\end{tabular}

\section{Conclusiones}

Llegados a este punto del análisis nos preguntamos si vale la pena realizar una nueva edición filológica crítica de las cartas. No la creemos necesaria, consideramos muy provechosas las ediciones de Gachard y de Bouza, diferentes entre sí, pero encomiables ambas: respetuosa del texto la una, actualizada pero rica en detalles y notas históricos, la otra.

Sin embargo, echamos de menos -desde la perspectiva de la filología-, más claridad en los criterios de edición.

Asimismo, consideramos imprescindible, cara a posteriores estudios, dar a conocer la signatura topográfica exacta bajo la cual se en- 
cuentra dicha documentación. Este hecho, por insignificante que parezca, es una herramienta fundamental con la que cuenta el filólogo y, a su vez, es la mejor manera de proteger documentos de esta envergadura.

Sabíamos gracias a la edición de 1998 que estas cartas, a excepción de la número III, se encontraban en el Archivio di Stato di Torino señaladas como Lettere Principi Forestieri. Creímos, pues, oportuno contactar con la dirección del archivo turinés con el fin de que nos proporcionara la signatura exacta bajo la cual se halla esta documentación. La mayor parte de este corpus documental se encuentra en el Archivio de Stato di Torino como Lettere diverse Real Casa, Lettere Principi Forestieri, Lettere di Filippo II d'Asburgo - mazzi 96-97, distribuidas de la siguiente manera:

- cartas I-XXVIII: mazzo 96.

- cartas XXIX-XXXIII: mazzo 97.

La carta III no se halla en el Archivio di Stato di Torino sino en el $\mathrm{Mu}$ seo Storico, bajo la inscripción: Autografi di huomini illustri-Spagna.

Lamentablemente no nos ha sido proporcionada la localización exacta de las cartas V, VI, VIII, X, XI y XXXIV.

\section{Bibliografía}

Altadonna, G. 1986. Cartas de Felipe II a Carlos Manuel II, duque de Saboya (1583-1596). Cuadernos de investigación histórica 9: 137-190.

Bouza, F. (ed.). 1998. Cartas de Felipe II a sus hijas. Madrid: Ediciones Akal. Castronovo, V. 1977. Carlo Emanuele I di Savoia. Dizionario Biografico degli Italiani. Roma, vol. 20, 326-340.

Gachard, L. P. 1884. Lettres de Philippe II a ses filles les Infantes Isabelle et Catherine: écrites pendant son voyage en Portugal (1581-1583). París: Librairie Plon.

Portillo Díaz, L. E. 1943[?]. Cartas de Felipe II a sus hijas. Madrid: Ediciones Lepanto.

Pérez de Guzmán, J. 1876. El lado amable de un rey severo. Revista Contemporánea 6: 76-91. Madrid.

Spivakovsky, E. 1975. Felipe II. Epistolario familiar. Cartas a su hija, la infanta doña Catalina (1585-1596). Madrid: Espasa Calpe. 
